\title{
Claudio Simon Hutz: Pesquisador e Líder Positivo da Avaliação Psicológica Brasileira
}

\author{
Claudia Hofheinz Giacomoni ${ }^{1}$ \\ ${ }^{1}$ Universidade Federal do Rio Grande do Sul, RS, Brasil
}

\begin{abstract}
RESUMO: O psicólogo, professor e pesquisador da área da avaliação psicológica, Claudio Simon Hutz, é homenageado neste artigo. O objetivo foi apresentar a sua biografia e toda a sua contribuição para com a psicologia, e mais, especificamente, a área da avaliação psicológica até o presente momento. São revelados aspectos sobre a sua história de vida profissional docente e acadêmica, a sua atuação na pós-graduação stricto e lato sensue seu perfil como gestor. Além disso, são resgatadas as suas participações em entidades profissionais e científicas e realizado o mapeamento da sua produção científica. O professor Claudio Simon Hutz foi um pioneiro e é uma liderança da área da avaliação psicológica. Ao longo da sua trajetória, aqui descrita, primou pelo avanço da Psicologia científica brasileira e de uma prática profissional pautada pela ética e excelência.
\end{abstract}

Palavras-Chave: Avaliação Psicológica, Liderança, Biografia.

\section{Claudio Simon Hutz: Researcher and Positive Leader of the Brazilian Psychological Assessment}

\begin{abstract}
The psychologist, professor and researcher in the field of psychological assessment Claudio Simon Hutz is honored in this article. The objective was to present his biography and all his contributions to psychology, and more specifically, to the area of psychological until today. It reveals aspects about his professional and academic life, his work at the graduate and undergraduate schools and his profile as a manager. In addition, his participation in professional and scientific entities is tackled as well as his scientific production is mapped. Professor Claudio Simon Hutz was a pioneer and is a leader in the field of psychological assessment. Throughout his career, described here, he excelled in the advancement of Brazilian scientific psychology and of a professional practice based on ethics and excellence.
\end{abstract}

Keywords: Psychological Evaluation, Leadership, Biography.

\section{Claudio Simon Hutz: Investigador y Líder Positivo de la Evaluación Psicológica Brasileña}

RESUMEN: El psicólogo, profesor e investigador del área de la evaluación psicológica, Claudio Simon Hutz, es homenajeado en este artículo. El objetivo fue presentar su biografía y toda su contribución a la psicología, y más específicamente, el área de la evaluación psicológica hasta el presente momento. Se revelan aspectos sobre su historia de vida profesional docente y académica, su actuación en el postgrado stricto y lato sensu y su perfil como gestor. Además, son rescatadas sus participaciones en entidades profesionales y científicas, y realizado el mapeo de su producción científica. El profesor Claudio Simon Hutz fue un pionero y es un liderazgo del área de la evaluación psicológica. A lo largo de su trayectoria, aquí descrita, primó por el avance de la Psicología científica brasileña y de una práctica profesional pautada por la ética y la excelencia.

Palabras-clave: Evaluación Psicológica, Liderazgo, Biografía. 
Se existe, pode ser medido! (frase escrita no Laboratório de Mensuração, UFRGS).

Na placa entregue ao professor Claudio Hutz em razão de sua aposentadoria lê-se: "Difícil será encontrarmos uma métrica para o tamanho da tua colaboração e dedicação ao Programa de Pós-Graduação em Psicologia - UFRGS. O que temos certeza é que o tamanho de efeito é enorme!”.

Escrever sobre o pesquisador, cientista e professor Claudio Simon Hutz é uma tarefa prazerosa e desafiadora. Trata-se de abordar aspectos de sua vida, história, contribuições científicas, seu impacto como formador de recursos humanos, os efeitos de seus empreendimentos para a área de conhecimento e para a comunidade científica, bem como para a prática profissional, ou seja, sobre o seu legado. Atendi a incumbência de preparar este artigo ciente da grande responsabilidade, pois trata-se de um orientado homenageando o orientador, hoje colega de trabalho.

\section{Biografia}

Claudio Simon Hutz nasceu em Porto Alegre (RS), em 2 de outubro de 1948, filho de Arhon Hutz e Silvia Collen Hutz. O pai, médico endocrinologista, foi professor da Faculdade de Medicina da Universidade Federal do Rio Grande do Sul (UFRGS) ao longo de toda a vida profissional. Presidiu a divisão gaúcha da Sociedade Brasileira de Endocrinologia e integrou comissões científicas de vários eventos de sua área. A mãe atuou no Laboratório de Análises Clínicas que a família manteve até a década de 1960-1970, tendo também trabalhado junto ao marido. Claudio, o filho mais velho, possui duas irmãs. Mara Helena Hutz, bióloga, doutora em genética e biologia molecular, pesquisadora 1A do Conselho Nacional de Desenvolvimento Científico e Tecnológico (CNPq), premiada e membro titular da Academia Brasileira de Ciências, é professora titular do Departamento de Genética da UFRGS. Rosane Hutz vive no México há muitos anos; lecionou português em programas de extensão da Universidade Autônoma do México. Atualmente, trabalha no consulado brasileiro e é tradutora juramentada de português para espanhol e espanhol para português.

Conforme relatam pessoas próximas, o adolescente Claudio foi um aluno inteligente e inquieto. Participou ativamente de política estudantil, em especial, durante o período do ensino ginasial. Presidente do grêmio estudantil de sua escola, o Colégio Estadual Júlio de Castilhos (escola pública centenária em Porto Alegre), sofreu os efeitos de sua atuação e liderança naqueles anos difíceis: foi expulso da escola a poucos meses de finalizar os estudos. A matrícula em outras escolas tornou-se difícil devido ao histórico de atividades na política estudantil. O pai conseguiu matriculá-lo no Colégio Ruy Barbosa, cujo diretor era o médico Saul Nicolaiewsky. Após concluir o curso científico frequentou dois anos do curso de Ciências Econômicas da UFRGS. Nesse mesmo período, realizou o serviço militar no Centro de Preparação de Oficiais da Reserva (CPOR).

Em 1969, por decisão própria e com o apoio da família, foi estudar em Israel. Na Universidade de Haifa, no período de quatro anos, realizou o curso de graduação em Psicologia. Em setembro de 1973 retornou ao Brasil conforme o planejado, coincidentemente uma semana antes do início da guerra do Yom Kippur. Ao retornar ao Rio Grande do Sul, realizou o processo de revalidação do seu diploma cumprindo complementação de carga horária e estágios na Pontifícia Universidade Católica do Rio Grande do Sul (PUC-RS). Possui duas filhas: Aida Hutz-Midgett, psicóloga, professora no Department of Counselor Education, na Boise State University, em Idaho (EUA), e Ariela Kohler Hutz, bacharel em administração e em ciências contábeis, controladoria e finanças, atualmente realizando curso de pós-graduação em Berlim (Alemanha).

\section{Vida profissional docente e acadêmica}

Em 1973, recém-chegado de Israel, Claudio foi convidado pela professora Jurema Alcides Cunha, referência na área da Avaliação Psicológica brasileira e integrante do grupo de professores fundadores do Curso de Psicologia da Universidade do Vale do Rio dos Sinos (Unisinos), a participar do corpo docente do novo curso. O curso da Unisinos recebeu a sua primeira turma em 1972, sendo o segundo voltado a formação em Psicologia do Estado (o primeiro foi o curso da PUC-RS, fundado em 1962).

Segundo Gomes (2009), o convite de Cunha constituiu-se em marco na vida de Hutz. Em dois sentidos: a opção pela carreira universitária e, nesta, o interesse pelas investigações em avaliação psicológica. No ano de 1975, foi integrado também ao Departamento de Psicologia do Instituto de Filosofia e Ciências Humanas (IFCH) da UFRGS. Nesse período inicial, teve também experiência profissional fora da academia, 
tendo atuado atuou como psicólogo de uma grande empresa em Porto Alegre.

Nos Estados Unidos dedicou-se aos estudos de pós-graduação. Entre 1977 e 1979 realizou o curso de mestrado na Universidade de Iowa. Orientado pelo professor Harold P. Bechtoldt, elaborou a dissertação The Development of Binocular Vision in Infants. Em 1981, na mesma universidade, concluiu o curso de doutorado, elaborando a tese The Effect of Belief in Premises and Conclusions on Children's Logical Reasoning sob a orientação do professor Gordon Cantor. Retornou ao Brasil no final de 1981, reintegrando-se à UFRGS. Em 1982, passou a atuar também no curso de Psicologia da PUC-RS. Nos anos seguintes, o professor Hutz atuou intensivamente na consolidação dos cursos de Psicologia das duas instituições.

\section{Atuação na pós-graduação stricto e lato sensu}

Entre os anos de 1982 e 1987, o professor Hutz, juntamente com o professor William Barbosa Gomes, integrou o corpo docente do Programa de Pós-Graduação da PUC-RS. O Programa era coordenado pela professora Juracy C. Marques. Naquele programa, teve os seus primeiros orientandos. Paralelamente, na UFRGS, participou da criação da Revista Psicologia: Reflexão e Crítica, no ano de 1986, novamente com a parceria do professor William Barbosa Gomes, o primeiro editor-geral. O ano de 1988, marca a sua opção pela dedicação exclusiva a Universidade Federal do Rio Grande do Sul.

Quinze anos após o início do Curso de Psicologia da UFRGS, surgia o Programa de Pós-graduação (PPG) em Psicologia do Desenvolvimento, hoje denominado PPG-Psicologia. Conforme Piccinini, Palombini, Gauer, Machado, e Simanke (2014), a criação do Programa teve inúmeros impactos no Instituto de Psicologia da UFRGS, não só por ser o primeiro, mas pela sua proposta inovadora, com destaque para a interação graduação e pós-graduação, tanto na inserção dos pós-graduandos nas atividades de ensino e extensão, como no envolvimento de graduandos com as pesquisas da pós-graduação. Um pequeno grupo de professores, todos doutores recém-chegados de fora do país - Claudio Hutz, William Barbosa Gomes, Ângela Biaggio, Tânia Sperb e César Piccinini -, foi responsável pela construção da proposta e consolidação desse PPG que completa três décadas, conjuntamente com a colaboração dos professores que ao longo do tempo se integraram ao Departamento de Psicologia da Personalidade e do Desenvolvimento. Há quatro triênios consecutivos, o programa tem obtido o conceito sete, avaliação máxima na Coordenação de Aperfeiçoamento de Pessoal de Nível Superior (Capes).

Entre os anos de 1992 e 1993, o professor Hutz realizou o pós-doutoramento na Arizona State University (EUA). Em 1996, tornou-se professor titular da UFRGS, através de concurso público.

A partir do ano de 2000, os primeiros cursos de especialização em temas de Psicologia em nível lato sensu do Instituto de Psicologia da UFRGS foram impulsionados pelo trabalho dedicado do professor Claudio. Cursos de especialização em Saúde Comunitária, Avaliação Psicológica, Psicologia Hospitalar e Transtornos do Desenvolvimento passaram a ser organizados regularmente. Nos anos seguintes, novos cursos passaram a ser ofertados: Infância e Família, Neuropsicologia, Terapia Cognitivo Comportamental, Orientação Profissional, Psicologia Escolar, Psicologia Organizacional e Psicologia Positiva. Até o presente momento, foram formados aproximadamente um milhar de especialistas. Durante todos esses anos, a coordenação do setor de gerenciamento de todos esses cursos de especialização esteve a cargo do professor Claudio.

\section{Gestor}

Ao longo da carreira, Claudio Hutz ocupou quase todos os cargos gestores da Universidade. Foi chefe de departamento em vários mandatos. Foi coordenador do Curso de Psicologia, coordenador do Programa de Pós-Graduação. Durante o quadriênio 1998-2002, foi diretor do Instituto de Psicologia da UFRGS. Foi o representante da área das Ciências Humanas na Comissão Máxima da Universidade que tratava do financiamento da Iniciação Científica. Foi membro dos Comitês de Pesquisa, de Extensão, de Ética, entre outros.

\section{Participação em entidades profissionais e científicas}

Desde o início da sua vida profissional-acadêmica, Hutz foi uma liderança no desenvolvimento de entidades científicas, bem como, de entidades profissionais. Participou da gestão do Conselho Regional de Psicologia n.07 entre os anos de 1983-1986. Após ter sido secretário da Associação Nacional de Pesquisa e Pós-Graduação em Psicologia (Anpepp), na gestão 1988-1990, foi vice- 
-presidente na gestão 1996-1998 e presidente no biênio 1998-2000. Integrou, com os professores Luiz Pasquali e Solange Wechsler, o grupo de trabalho de Avaliação Psicológica da Anpepp que gestou o Instituto Brasileiro de Avaliação Psicológica (IBAP). Foi Presidente do IBAP no mandato 2003-2005. Mais recentemente, foi o fundador e primeiro presidente da Associação Brasileira de Psicologia Positiva (2013-2018).

Esteve envolvido com a organização e promoção dos principais eventos de Avaliação Psicológica no Brasil. Na década de 1990, juntamente com os professores de avaliação psicológica gaúcha promoveu os Encontros de Testes. Após a fundação do IBAP participou das comissões dos eventos nacionais de avaliação psicológica: I Congresso Nacional de Avaliação Psicológica (2003), II, III, V e VI Congresso Brasileiro de Avaliação Psicológica $(2005,2007,2011,2013)$.

No ano de 2002, juntamente com Ana Mercês Bahia Bock, coordenou a organização do I Congresso Brasileiro Psicologia: Ciência e Profissão, realizado de 1 a 5 de setembro, na Cidade Universitária da Universidade de São Paulo (USP), em São Paulo. O Congresso resultou de debates no âmbito do Fórum de Entidades Nacionais Psicologia Brasileira. Nos Anais publicados do Congresso lê-se:

A alavanca mestra que move o 'Ciência e Profissão’ é a retomada do elo perdido entre a ciência e a profissão, isto é, o evento deverá significar o início da reconstrução da ponte entre a pesquisa e a prática profissional. A finalidade é que a Psicologia no Brasil tenha sua ciência alimentada pelas indagações que nascem na prática da profissão, ao mesmo tempo em que os psicólogos tenham um amplo acesso aos conhecimentos científicos.

O evento contou com em torno de dez mil participantes.

Presidiu o I e o II Congresso Brasileiro de Psicologia Positiva, respectivamente nos anos de 2014 e 2016. Como presidente, promoveu a vinda para o Brasil, do principal evento Internacional sobre Personalidade, o $2^{\text {nd }}$ World Conference on Personality, no início de 2016. Participou em comissões científicas das principais agências de fomento nacionais: Capes, CNPq, Instituto Nacional de Estudos e Pesquisas Educacionais Anísio Teixeira (INEP), Fundação de Amparo à Pesquisa do Estado do Rio Grande do Sul (Fapergs) e Fundação de Amparo à Pesquisa do Estado de São Paulo (Fapesp).

\section{Pesquisador e produção científica}

Claudio Hutz, ao longo de sua trajetória acadêmica, produziu mais de 200 artigos científicos publicados em periódicos nacionais e internacionais. Publicou mais de 30 livros em Psicologia e mais de 50 capítulos, a sua maioria na área da avaliação psicológica. No ano de 2011 recebeu o Prêmio Pesquisador Gaúcho, importante reconhecimento da comunidade científica gaúcha, patrocinado pela Fapergs. É pesquisador 1A do CNPq. Coordena o Laboratório de Mensuração da UFRGS. Suas linhas de pesquisa são nas áreas da Psicologia Positiva, Desenvolvimento Social e da Personalidade e Avaliação Psicológica e Psicometria (com ênfase na construção e validação de instrumentos para a avaliação da personalidade). Produziu instrumentos de avaliação psicológica de excelência que contribuem muito para a prática profissional, com destaque, entre outros, para a Bateria Fatorial da Personalidade (BFP).

\section{Professor e orientador acadêmico}

Ao longo de sua trajetória como docente orientou 45 dissertações de mestrado e 23 teses de doutorado. Permanece como professor permanente do PPG Psicologia da UFRGS, orientando alunos da pós-graduação e de iniciação científica. Em vários momentos ouvimos o professor Claudio Hutz afirmar "a melhor maneira de escolher/conhecer um orientador é verificar por onde andam os seus ex-orientandos". Seu papel como formador de pesquisadores e professores pode ser observado na quantidade de ex-orientandos que hoje integram vários programas de pós-graduação em diferentes regiões do Brasil.

No Curso de Psicologia da UFRGS ministrou entre outras as disciplinas de Psicologia da Personalidade, Técnicas Psicométricas, Pesquisa em Psicologia, Avaliação Psicológica. No Programa de Pós-Graduação em Psicologia ministra há alguns anos a disciplina intitulada Prática e Produção Científica em Psicologia que estimula a produção científica em parceria com colegas e a apreciação crítica dos materiais produzidos através da emissão de pareceres. Tem por objetivo facilitar o desenvolvimento de habilidades e competências que pesquisadores autônomos requerem para realizar estudos em equipe, divulgar adequadamente sua produção científica e emitir pareceres. A estimulação para autonomia e liderança são características muito presentes na relação orientador-orientando e professor-aluno. 


\section{Homenagem}

Ao longo da carreira de excelência do professor-pesquisador Claudio Simon Hutz, observamos seu pioneirismo, competência e importância para a área da Avaliação Psicológica brasileira. Sua trajetória se entrelaça com a história da Pós-Graduação Brasileira em Psicologia, bem como com a história do ensino e pesquisa da Psicologia da UFRGS. Contribuiu para que se estabelecessem as bases de um PPG de excelência e de uma graduação em Psicologia de destaque.

Brinda-nos sempre com a visão de uma universidade moderna e de um programa de pós-graduação que pode sempre se superar em qualidade. Primou pela interação entre a graduação e a pós-graduação.
Mostrou ser um gestor inovador, criativo e motivador. Trabalhou em todos os cargos da universidade, indo além das atividades de pesquisa e ensino. Sua diversificada produção científica, de destaque nacional e internacional, é referência para todos na área. Além disso, exercita uma prática profissional pautada pela ética e excelência.

Representante exemplar de um grupo de pioneiros, o professor Claudio Hutz dedicou sua vida docente e de pesquisador para o desenvolvimento da Psicologia científica e para a formação de profissionais competentes. Nós e todos os que tiveram e têm a oportunidade de conviver com ele, resta-nos agradecer as suas contribuições, sua liderança positiva e rica convivência.

\section{Referências}

Gomes, W. (2009). A tradição em avaliação psicológica no Rio Grande do Sul: a liderança e a referência de Jurema Alcides Cunha. In: C. S. Hutz (Orgs.), Avanços e polêmicas em avaliação psicológica. São Paulo, SP: Casa do Psicólogo.

Hutz, C. S. (1979). The development of binocular vision in infants (Dissertação). University of Iowa, Iowa City, Estados Unidos.

Hutz, C. S. (1981). The effect of belief in premises and conclusions on children's logical reasoning (tese). University of Iowa, Iowa City, Estados Unidos.

Piccinini, C. A., Palombini, A., Gauer, G., Machado, P. S., \& Simanke, D. (2014). Curso de Psicologia da UFRGS 40 anos. Porto Alegre: Universidade Federal do Rio Grande do Sul.

\section{Cláudia Hofheinz Giacomoni}

Psicóloga. Mestre e Doutora em Psicologia pela UFRGS/YALE. Professora do Departamento de Psicologia do Desenvolvimento e da Personalidade e do Programa de Pós-Graduação em Psicologia da UFRGS. Coordenadora do Núcleo de Estudos em Psicologia Positiva (NEPP).

Endereço para Correspondência:

NEPP sala 124. Instituto de Psicologia UFRGS Av. Ramiro Barcelos, 2600. Porto Alegre RS

Recebido 06/02/2015

Reformulação 16/02/2017

Aprovado 14/03/2017

Received 02/06/2015

Reformulated $02 / 16 / 2017$

Approved 03/14/2017

Recebido 06/02/2015

Reformulado 16/02/2017

Aceptado 14/03/2017 
Psicologia: Ciência e Profissão 2018 v. 38 (núm. esp.), 189-194.

Como citar: Giacomoni, C. H. (2018). Claudio Simon Hutz: pesquisador e líder positivo da Avaliação Psicológica Brasileira. Psicologia: Ciência e Profissão, 38(n.spe), 189-194. https://doi.org/10.1590/1982-3703000208989

How to cite: Giacomoni, C. H. (2018). Claudio Simon Hutz: researcher and positive leader of the Brazilian Psychological Assessment. Psicologia: Ciência e Profissão, 38(n.spe), 189-194. https://doi.org/10.1590/1982-3703000208989

Cómo citar: Giacomoni, C. H. (2018). Claudio Simon Hutz: investigador y líder positivo de la Evaluación PsicológicaBrasileña.Psicologia:CiênciaeProfissão,38(n.spe),189-194.https://doi.org/10.1590/1982-3703000208989 